\title{
PRODUÇÃO DE MAMOGRAFIAS NO ÂMBITO DO SISTEMA ÚNICO DE SAÚDE
}

\section{Franciely Santos Kuhn}

Tecnóloga em Radiologia. Faculdade Estácio de Feira de Santana, Brasil.

\section{Amália Ivine Costa Santana}

Enfermeira. Mestra em Saúde Coletiva. Docente da Faculdade Estácio Feira de Santana, Brasil.

\section{Magno Conceição das Mercês}

Biólogo. Enfermeiro. Mestre em Saúde Coletiva. Docente na Universidade do Estado da Bahia (UNEB), Brasil.
RESUMO: Entre as modalidades de atenção previstas para o controle do câncer de mama está a detecção precoce pela realização de mamografia. O objetivo do estudo foi caracterizar segundo informações das usuárias e do exame a produção de mamografias no âmbito do Sistema Único de Saúde. Trata-se de estudo transversal descritivo acerca dos registros das mamografias realizadas no território brasileiro entre 2009 e 2014, cujas informações constavam no Sistema de Informação do Controle do Câncer de Mama. Ficou evidenciada baixa cobertura na região Norte do Brasil. Observou-se percentual maior de realização para as mulheres brancas e que tinham ensino fundamental incompleto. Em relação às categorias do Breast Imaging Reporting and Data System, os achados benignos foram os mais recorrentes entre as mulheres brasileiras. A recomendação de conduta mais frequente foi realização de mamografia em dois anos. O conhecimento de variáveis sociodemográficas no rastreamento de câncer de mama pode identificar grupos populacionais de risco para a realização de tal prática.

PALAVRAS-CHAVE: Mamografia; Sistema Único de Saúde; Câncer de mama.

\section{PRODUCTION OF MAMMOGRAMS WITHIN THE NATIONAL HEALTH SERVICE}

ABSTRACT: Early detection of breast cancer by mammography is one of the care modalities for the control of breast cancer. Current transversal and descriptive study analyzes the production of mammograms within the NHS, following information by patients and tests, and investigates the registration of mammograms in Brazil between 2009 and 2014. Information was obtained from the Information System for the Control of Breast Cancer. Low covering rate occurred in northern Brazil. A higher percentage was evidenced for white females with incomplete basic schooling. With regard to categories within the Breast Imaging Reporting and Data System, benign results were high among Brazilian females. More frequent behavior within a two-year period was recommended. Knowledge on socio-demographic variables in the screening of breast cancer may identify risk groups for the undertaking of such procedure.

KEY WORDS: Mammogram; National Health Service; Breast Cancer. 


\section{INTRODUÇÃO}

O câncer de mama é uma doença resultante da multiplicação de células anormais das mamas, tornando-se um dos mais temíveis entre as mulheres, afetando-as tanto psicologicamente como fisicamente. A detecção precoce tem como premissa impedir consequências desastrosas, e esse fato tornou-se o objetivo dos programas de saúde no Brasil $^{1}$. Excluídos os tumores de pele não melanoma, o câncer de mama é o mais incidente em mulheres de todas as regióes, exceto na região Norte do Brasil, onde o câncer de colo de útero ocupa primeira posição. Para o ano de 2016 foram estimados 57.960 casos novos de câncer de mama no país, que representam uma taxa de incidência de 56,2 casos a cada 100 mil mulheres. A incidência do câncer de mama tende a crescer progressivamente a partir dos 45 anos $^{2}$.

Com base nesses dados, as mulheres necessitam ter o conhecimento sobre a prevenção e detecção precoce do câncer de mama, com garantia de acesso a exames de rastreamento com qualidade, para que, caso se evidencie algum tipo de lesão palpável, obtenha esclarecimento imediato sobre o diagnóstico e, consequentemente, um tratamento adequado 3 .

A Agência Internacional para Pesquisa do Câncer (IARC), da Organização Mundial de Saúde (OMS), alerta que, para o efetivo controle do câncer de mama, é preciso que sejam desenvolvias ações intersetoriais capazes de promover e organizar ampla cadeia de atenção à mulher, que vai desde a informação em saúde, passando pela promoção de uma vida saudável (com identificação e combate aos riscos evitáveis), até o acesso à detecção precoce e ao tratamento oportuno e adequado para a doença em todas as suas fases evolutivas ${ }^{4}$.

Nesse aspecto, em 2008, foi assinada a lei 11.664/08, que determina, entre outras medidas, a realização de mamografia para todas as mulheres a partir dos 40 anos de idade pelo SUS. A publicação da lei parte da premissa de que quanto mais cedo o câncer de mama é diagnosticado, maiores são as chances de cura e, consequentemente, de preservar as vidas das mulheres brasileiras 5 .

No ano seguinte, o Ministério da Saúde implantou o Sistema de Informação do Controle do Câncer de
Mama (SISMAMA), que é composto pelo módulo do prestador, utilizado pelos serviços de radiologia mamária e patologia para cadastro dos exames, emissão de laudos e avaliação de desempenho dos serviços; e pelo módulo de coordenação, utilizado pelos gestores estadual, regional e municipal para gerenciamento das ações de detecção precoce do câncer de mama e seguimento das mulheres com exames alterados. O SISMAMA tem o objetivo de padronizar os laudos e evitar desvios nas interpretações mamográficas e por esse motivo adota o Breast Imaging Reportingand Data System (BI-RADS), um sistema que expressa os achados mamográficos em categorias e propõe condutas clínicas de acordo com sua categorização, tornando a transmissão de dados do Sistema Único de Saúde (SUS) para o Ministério da Saúde mais rápido, fácil e fidedigno ${ }^{6}$. Nesse sentido, o objetivo deste trabalho foi caracterizar, segundo informações das usuárias e do exame, a produção de mamografias no âmbito do SUS, no período de 2009 à 2014.

\section{METODOLOGIA}

Trata-se de estudo de corte transversal de cunho descritivo acerca dos registros de realização das mamografias no Sistema de Informação do Controle do Câncer de Mama (SISMAMA). Este sistema é alimentado pelos municípios e Estados do Brasil, após realização de mamografias por serviços que executam os procedimentos referentes ao rastreamento do câncer de mama ${ }^{7}$.

A amostra do estudo incluiu todas as mamografias realizadas no âmbito do SUS e registradas no SISMAMA, entre 2009 e 2014 . O período de 2009 a 2014 foi escolhido pela disponibilidade dos dados de acesso ao público.

$\mathrm{O}$ instrumento que permitiu a análise dos dados compõe-se de quatro formulários de coleta de dados que alimentam o SISMAMA. Esses formulários são: a requisição de mamografia; o resultado de mamografia; a requisição de exame citopatológico e requisição de exame histopatológico. No presente estudo, em particular, foram utilizadas informações acerca dos dois primeiros formulários que compõem o sistema.

As variáveis selecionadas foram as características das mamografias (ano de realização, região prestadora, 
indicação clínica), características sociodemográficas (faixa etária, sexo, raça/cor, escolaridade), dados da anamnese (risco elevado para câncer de mama, realização de mamografia anterior), características relacionadas ao diagnóstico (recomendações de conduta, categoria BIRADs).

Os dados foram processados no Microsoft Office Excel 2007. Realizou-se a análise estatística descritiva por meio de frequências absoluta e relativa. Por se tratar de dados de domínio público on-line e que não permite a identificação dos indivíduos dispensou a apreciação pelo Comitê de Ética em Pesquisa.

\section{RESULTADOS}

No período em estudo foram realizadas 14.239.937 mamografias em todo o território brasileiro. Houve variação na realização das mamografias no período estudado, sendo que no ano de 2012 realizou-se o maior número de exames, representando $24,8 \%$ do total. As regiões prestadoras apresentaram diferenças entre as frequências de notificação, sendo as maiores nas regiões Sudeste $(56,5 \%)$ e Nordeste $(19,3 \%)$; a região Norte apresentou a menor ocorrência, com 3,2\% de realizações no período. Na Figura 1 é apresentada a realização dos exames por regiões do Brasil.

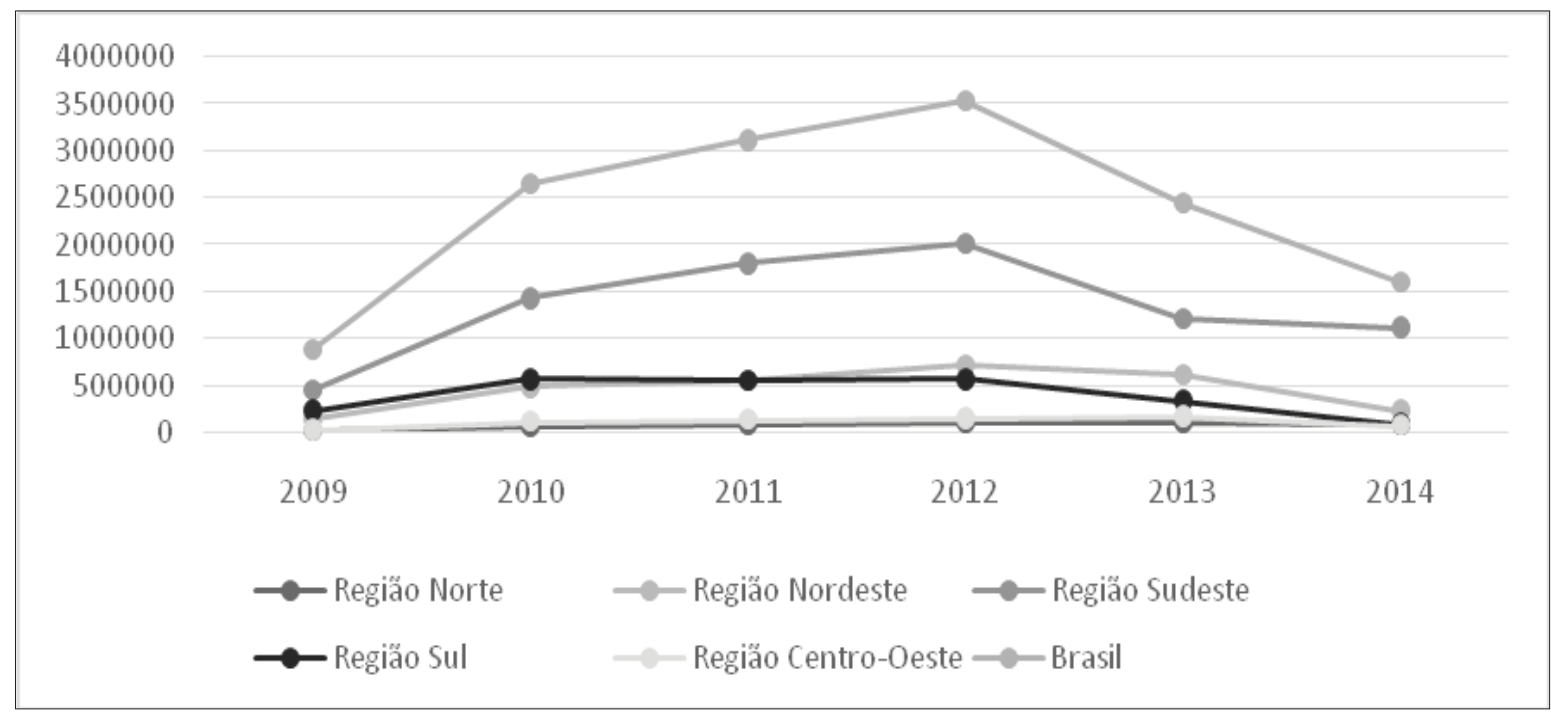

Figura 1. Realização de mamografias por região prestadora, Brasil, 2009-2014.

Fonte: SISMAMA, 2009-2014.

Em relação às características sociodemográficas, a maioria dos exames foi realizada entre pessoas de 40 e 59 anos (69,9\%), do sexo feminino (99,9\%), com até o ensino fundamental incompleto $(51,8 \%)$ e da raça/cor da pele branca (54,67\%). Para essa variável, em particular, não havia informação para $58,0 \%$ dos casos. No que dizia respeito ao tempo de realização de mamografia anterior, notou-se que a maioria das usuárias submeteuse ao exame com o intervalo de um ano (43,0\%). Um percentual significativo representou mulheres que realizaram o exame a cada dois anos (Figura 2). Percebeuse que o tempo de realização da mamografia anterior foi maior na região Centro-Oeste, onde 7,8\% das mulheres informaram ter realizado a última mamografia há quatro anos ou mais.

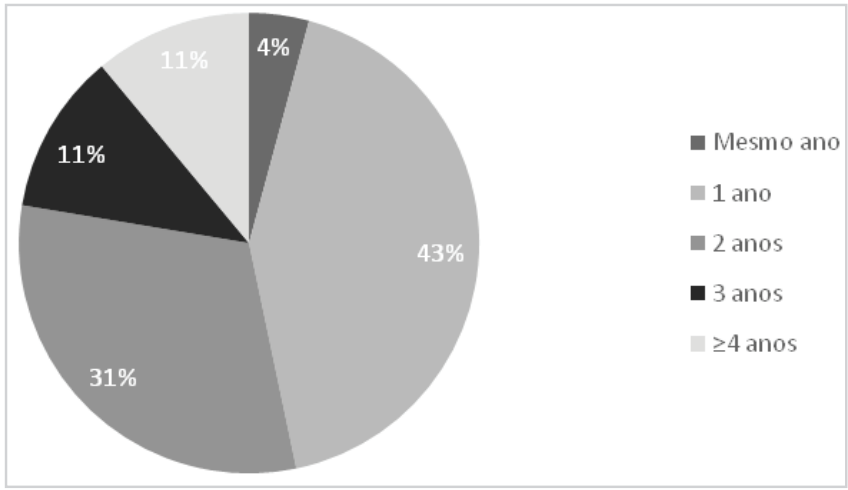

Figura 2. Realização de mamografia anterior, Brasil, 2009-2014. Fonte: SISMAMA, 2009-2014. 
Quanto à indicação clínica, em 95,6\% dos casos, foi realizada a mamografia de rastreamento. Sobre o risco elevado para câncer de mama, $87,3 \%$ das pessoas referiram não possuir esse tipo de risco. Em relação às características do diagnóstico, os achados mais frequentes do sistema BIRADs foi a categoria 2 (achados mamográficos benignos) e a categoria 1 (sem achados mamográficos) com 43,0\% e 41,0\% das ocorrências, respectivamente (Figura 3). A recomendação de conduta mais frequente foi a realização de mamografia em dois anos $(84,5 \%)$.

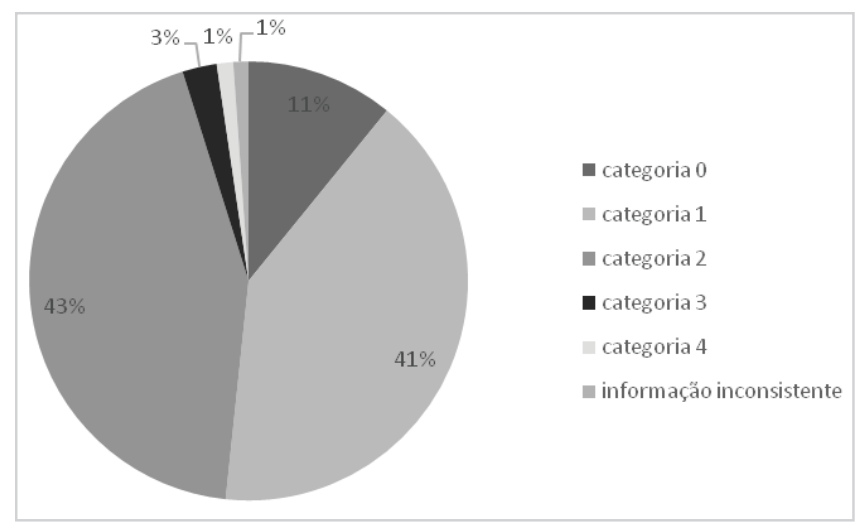

Figura3. Categorias do sistema BI-RADs, Brasil, 2009-2014. Fonte: SISMAMA, 2009-2014.

\section{DISCUSSÃO}

Esse estudo demonstrou que o Sudeste é a região que mais realiza mamografias no Brasil. Segundo o Instituto Brasileiro de Geografia e Estatística, ${ }^{8}$ a região Sudeste agrega a maior concentração da população brasileira, o que pode explicar o achado. Além disso, estudo conduzido por Oliveira et $\mathrm{al}^{9}$ apontou que residir em área metropolitana aumenta em três vezes a chance de realizar mamografia, fato que está intimamente relacionado à maior oferta de serviços de saúde mais numerosos e complexos.

Por outro lado, o Norte foi a região onde foram realizadas apenas 3,2\% de mamografias. Esse achado pode estar associado ao fato de que o Norte é a região com a menor densidade populacional do $\mathrm{Brasil}^{8}$. Existe uma particularidade que também merece consideração nesse aspecto, conforme destaca Xavier et $\mathrm{al}^{10}$, ao ponderarem a dificuldade de realização de mamografias atrelada às distâncias geográficas. No entanto, a despeito dessas informações, estudo evidenciou pior avaliação da região
Norte em relação ao estado de saúde da população, maior restrição de atividades de saúde realizadas e menor uso de serviços de saúde na região ${ }^{11}$.

Segundo Xavier et al, ${ }^{10}$ somente cinco Estados brasileiros (Minas Gerais, Espírito Santo, São Paulo, Santa Catarina e Paraná) realizaram exames de mamografias acima de 50,0\% da necessidade de suas populações. A cobertura de exames foi ainda menor na região Norte, onde apenas $18,0 \%$ da necessidade de exames da sua população foram atendidas, fortalecendo os dados aqui encontrados.

Em relação à idade, a União Europeia recomenda o exame para as mulheres de 50 a 69 anos, parâmetro adotado pela maior parte dos Estados-Membros. O intervalo entre os exames é de dois anos, exceto no Reino Unido e em Malta, que adotam intervalo de três anos. No ano 2000, na Argentina, a recomendação era de exame bianual na faixa de 50 a 70 anos, no Chile, trianual na faixa de 50 a 64, e anual para esta mesma faixa no Uruguai. No Brasil, desde 2004, o rastreamento por mamografia é recomendado pelo Ministério da Saúde para as mulheres entre 50 e 69 anos, a cada dois anos, e a Sociedade Brasileira de Mastologia recomenda a mamografia anual a partir dos 40 anos?.

A recomendação no Brasil, atualizada em 2015, é que mulheres entre 50 e 69 anos se submetam a uma mamografia a cada dois anos. Essa é também a rotina adotada na maior parte dos países que implantaram o rastreamento do câncer de mama e tiveram impacto na redução da mortalidade por essa doença ${ }^{2}$. No presente estudo, a maioria das mulheres realizou a mamografia entre 40 a 59 anos, demonstrando assim que as recomendações do Ministério da Saúde estão sendo contempladas.

O câncer de mama é o mais comum entre as mulheres no mundo e no Brasil, depois do de pele não melanoma, respondendo por cerca de $25 \%$ dos casos novos a cada ano. $O$ câncer de mama também acomete homens, porém é raro, representando apenas $1 \%$ do total de casos da doença ${ }^{2}$. O câncer de mama é mais comum em mulheres por diversos fatores como a menarca precoce, primeira gravidez após os 30 anos, reposição hormonal pós-menopausa principalmente se prolongar por mais de cinco anos, entre outros fatores ${ }^{12}$. Assim sendo, o 
Ministério da Saúde preconiza a realização periódica do exame apenas para a população feminina, o que explica a realização quase absoluta entre mulheres.

Sobre a escolaridade, percebeu-se que a maioria das pessoas que realizou a mamografia no âmbito do SUS tinha ensino fundamental incompleto. Em contraposição a esse achado, Lages e colaboradores ${ }^{13}$ afirmam que a não realização das mamografias é menor quanto menor os níveis de escolaridade, ou seja, a condição social tem influência não só na percepção do risco e nos fatores comportamentais que influenciam a decisão de procurar o serviço, mas também é ponto determinante na acessibilidade à realização do exame. No entanto, o atendimento pelo SUS é mais procurado por usuários de baixa escolaridade e renda ${ }^{14,15}$, o que corrobora os achados do presente estudo.

Aqui, $54,0 \%$ das mulheres que realizaram mamografia eram da cor da pele branca. De acordo com Chor e colaboradores ${ }^{16}$, as mulheres negras e pardas tiveram chance $32,0 \%$ menor e $42,0 \%$ menor de realizar mamografia, respectivamente, quando comparadas às mulheres brancas no Brasil. Esses resultados sugerem que cor/raça pode constituir uma das barreiras para a realização de mamografia. Percebeu-se também nesse estudo que, em relação à variável raça/cor, houve perdas significativas no preenchimento. É importante ressaltar que a portaria $\mathrm{n}^{\circ} 719 / 07$, art. $1^{\circ}$, prevê "incluir o campo Raça/Cor, nos Sistemas de Informação Ambulatorial e Hospitalar, para efetivação do registro nos instrumento de coleta de dados de identificação do usuário do SUS"17. Nesse aspecto, percebe-se alta incompletitude dos dados e, portanto, pouca importância dada a essa variável pelos profissionais que preenchem o formulário.

Nesse estudo, a maioria das mulheres realizou mamografia com intervalo de um ano. Acredita-se que ações promovidas pelo Ministério da Saúde, tais como o Outubro Rosa, sejam responsáveis por viabilizar a realização desse exame com tal frequência entre as mulheres. Malta, Moura e Bernal ${ }^{18}$ afirmam que, no Brasil, $54,23 \%$ das mulheres de 59 a 69 anos se submeteram ao exame de mamografia nos últimos dois anos. O relatório evidenciou que a região Sudeste apresentou a maior proporção de realização nesse intervalo, 63,77\% e a Norte o resultado mais baixo, $35,26 \%$.
No presente estudo observou-se que na região Centro-oeste as mamografias apresentaram o maior intervalo de realização, com 7,8\% das mulheres informando ter se submetido à última mamografia há quatro anos ou mais. Em relação à periodicidade de realização da mamografia, o INCA ${ }^{2}$ aponta que o intervalo de dois anos constitui-se como a indicação mais segura atualmente, afinal, apesar de baixa, existe exposição à radiação, fator de risco para o câncer de mama. Ressalta ainda que apesar de pequeno, o risco é real, aumentando com a exposição repetida ao exame.

Sobre a indicação clínica, o SISMAMA apresentou dados positivos, em que 95,6\% das recomendações são de rastreamento, principal objetivo do exame. A detecção precoce do câncer de mama em estágio inicial por meio da mamografia é umas das maneiras efetivas de reduzir as taxas de morbidade e mortalidade por esta causa ${ }^{19}$. De acordo com Zimmermann et $\mathrm{al}^{20}$, a mamografia de rastreamento é considerada a mais sensível para a detecção precoce de câncer de mama. A mamografia de rastreamento evidencia-se muito significativa na redução das taxas de mortalidade.

Geralmente, o termo BI-RADS acompanha os laudos das mamografias, identificando possíveis focos de câncer, classificando-os de em seis categorias. A categoria 0 corresponde a uma mamografia inconclusiva, que indica serem necessários outros exames de imagem adicionais para estabelecer o diagnóstico. A categoria 1 corresponde a evidências de que a mama não apresenta indícios de alterações nem malignas nem benignas. A categoria 2 representa a mama que possui alguma alteração, porém na maiorias das vezes esse diagnóstico é benigno. A categoria 3 representa uma suspeita de alteração tecidual, porém não recomenda biópsia, mas repetição do exame em menor tempo de intervalo. A categoria 4 indica lesão suspeita para câncer e recomenda realização de biópsia. A categoria 5 corresponde a achados altamente sugestivos de malignidade, e por fim a categoria 6 reserva-se a pacientes com biópsia prévia e malignidade comprovada ${ }^{21}$.

$\mathrm{Na}$ presente investigação percebeu-se que a categoria 2 foi a mais recorrente $(43,0 \%)$, contrariando estudo realizado por Prado e Guerra ${ }^{22}$ no qual a categoria 4 apresentou maior ocorrência $(76,28 \%)$ em relação às outras categorias. Segundo Vieira e Toigo $^{23}$, a avaliação mamográfica, utilizando a classificação pelo método BIRADS, permite uma adequada padronização dos laudos, 
o que ajuda na comparação de exames por diferentes radiologistas e condutas a serem tomadas frente a achados mamográficos específicos, eliminando conflitos entre laudos de diferentes profissionais, bem como quanto à conduta médica a ser seguida. Esta classificação permite com segurança predizer que há benignidade quando enquadradas nas classes 1 e 2 . E a diminuta chance de malignidade quando na classe 3 . No entanto, vale ressaltar que o sistema BI-RADS considera alguns fatores de risco individuais importantes para definir o risco de malignidade ${ }^{24}$, fortalecendo a ideia de que essas mulheres merecem ser acompanhadas, sendo necessário estabelecer o elenco de fatores de risco pessoais para definição da conduta clínica a ser adotada.

\section{CONCLUSÃO}

O conhecimento de variáveis sociodemograficas no rastreamento de câncer de mama pode identificar grupos populacionais de risco para a realização de tal prática. O SUS é um grande financiador de realizações deste exame, mas ainda mostrou-se incapaz de suprir as necessidades existentes. Os dados encontrados nesse estudo reforçam a necessidade de implantação de ações para a população que enfrenta barreiras geográficas, organizacionais ou do serviço de saúde, socioculturais e econômicas.

O estabelecimento da relação entre produção de serviços de saúde e as características da população atendida apontam para estratégias de oferta e organização de serviços que possam corresponder aos determinantes sociais na perspectiva de superar iniquidades em saúde. Em última instância, o que se espera é construir um SUS cujos princípios e diretrizes possam ser vistos e sentidos na prática.

\section{REFERÊNCIAS}

1. Zeitune MM, Faria JCNM, Cruz AHS, Reis AAS, Santos RS. Relações genéticas e medidas socioeducativas para a prevenção dos carcinomas mamários. Saude e pesqui., 2013;6(2):287-93.

2. Instituto Nacional de Câncer [homepage na internet]. Incidências de câncer no Brasil. Estimativa 2016. [acesso em 05 de maio de 2017]. Disponível em: http://www.inca.gov.br/estimativa/2016/sintese-deresultados-comentarios.aspv.

3. Souza, FFS. (Vi)vendo e aprendendo: estudo com mulheres idosas sobre a prevenção do câncer de mama e colo de útero. Rio de Janeiro: Escola de Enfermagem Aurora de Afonso Costa, Universidade Federal Fluminense, 2016. Trabalho de Conclusão de Curso em Enfermagem e Licenciatura.

4. Gonçalves J, Siqueira ASE, Rocha IGA, Lima EFF, Alves LS, Silva BO, et al. Evolução histórica das politicas para o controle do câncer de mama no Brasil. Rev. saúde coletiva e bioética. 2016;8(1):1-23.

5. Brasil. Lei no $11.664 / 08$ de 29/04/2008. Dispõe sobre a efetivação de ações de saúde que assegurem a prevenção, a detecção, o tratamento e o seguimento dos canceres do colo uterino e de mama, no âmbito do sistema único de saúde SUS. Diário Oficial [da] República Federativa do Brasil. 29 abr 2008.

6. Santos SBL, Koch HA. Análise do Sistema de Informação do Programa de Controle do Câncer de Mama (SISMAMA) mediante avaliação de 1.000 exames nas cidades de Barra Mansa e Volta Redonda. Radiol Bras. 2010;43(5):295-301.

7. Brasil. Portaria $\mathrm{n}^{\mathrm{o}} 779$ de $31 / 12 / 2008$. Define como sistema de informação oficial do Ministério da Saúde, a ser utilizado para o fornecimento dos dados informatizados dos procedimentos relacionados ao rastreamento e a confirmação diagnóstica do câncer de mama, o Sistema de Informação do Controle do Câncer de Mama (SISMAMA). Diário Oficial [da] República Federativa do Brasil. 02 jan 2009.

8. Instituto Brasileiro de Geografia e Estatística [homepage na internet]. Censo demográfico 2011 [acesso em 27 mar 2005]. Disponível em: http:www. ibge.gov.br

9. Oliveira, EXG de; Pinheiro, RS; Melo, ECP; Carvalho, MS.Condicionantes socioeconômicos e geográficos do acesso à mamografia no Brasil, 2003-2008. Ciênc. saúde coletiva, 2011;16(9):3649-64. 
10. Xavier DR, Oliveira RAD, Matos VP, Viacava F, Carvalho CC. Cobertura de mamografias, alocação e uso de equipamentos nas Regiões de Saúde. Saúde debate, 2016;40(110):20-35.

11. Viacava F, Bellido JG. Health, access to services and sources of payment, according to household surveys. Ciênc. saúde coletiva, 2016;21(2):351-70.

12. Ohl ICB, Ohl RIB, Chavaglia SRR, Goldman RE. Ações públicas para o controle do câncer de mama no Brasil: revisão integrativa. Rev. Bras. Enferm. 2016;69(4):793-803.

13. Lages RB, Oliveira GP, Simeão Filho VM, Nogueira FM, Teles JBM, Vieira SC. Desigualdades associadas à não realização de mamografia na zona urbana de Teresina-Piauí-Brasil, 2010-2011. Rev. bras. epidemiol, 2012;15(4):737-47.

14. Ribeiro MCSA, Barata RB, Almeida MF de, Silva ZP. Perfil sociodemográfico e padrão de utilização de serviços de saúde para usuários e não-usuários do SUS - PNAD 2003. Ciênc. saúde coletiva, 2006;11(4):10111022.

15. Mazon LM, Negrelli MH. Satisfação dos usuários frente ao sistema único de saúde. Saúde Rev., 2016;16(43):45-51.

16. Chor D, Oliveira EXG, Melo ECP, Pinheiro RS, Carvalho MS. Desigualdade socioeconômica afeta a chance de realizar mamografia no Brasil. CMDSS 2011. [acesso em 05 de maio de 2016]. Disponível em: http:// cmdss2011.org/site/wp-content/uploads/2011/10/ CMDSS-1110.1v1.pdf

17. Brasil. Lei $\mathrm{n}^{\circ} 719 / 07$, Art. $1^{\circ}$. 28/12/2007. Incluir o campo Raça/Cor, nos Sistemas de Informação Ambulatorial e Hospitalar-(SIA/SIH/SUS), para efetivação do registro nos instrumento de coleta de dados de identificação do usuário do SUS. Diário Oficial [da] República Federativa do Brasil. 29 dez 2007.

18. Malta DC, Moura L, Bernal RTI. Differentials in risk factors for chronic non-communicable diseases from the race/color standpoint. Ciênc. saúde coletiva.
2015;20(3):713-25.

19. Santos GD dos, Chubaci RYS. O conhecimento sobre o câncer de mama e a mamografia das mulheres idosas frequentadoras de centros de convivência em São Paulo (SP, Brasil). Ciênc. saúde coletiva. 2011;16(5):2533-40.

20. Zimmermmann JB, Lima LM, Rabelo MV, Rabello PC, Martins RT, Peixoto TAG et al. Avaliação clínica, mamográfica e frequência de doenças mamárias em pacientes de um serviço privado de saúde. Clinical \& Biomedical Research. 2012;32(2):131-7.

21. Instituto Nacional de Câncer [homepage na internet]. Informações para o avanço das ações de prevenção do câncer de mama no Brasil. [acesso em 15 de junho de 2017]. Disponível em: http://bvsms.saude.gov.br/ bvs/publicacoes/inca/Sismama.pdf.

22. Prado GLM, Guerra MTPM. Valor preditivo positivo das categorias $3,4 \mathrm{e} 5$ do BreastImagingReportingand Data System (BI-RADS®). Radiol Bras. 2010;43(3):171-4.

23. Vieira AV, Toigo FT. Classificação BI-RADS ${ }^{\mathrm{TM}}$ : categorização de 4.968 mamografias. Radiol Bras. 2002;35(4):205-8.

24. Vasconcelos RG, Uemura R, Schirmbeck T, Vieira KM. Ultrassonografia mamária: aspectos contemporâneos. Com. Ciências Saúde, 2011;22(Sup 1):129-40.

Recebido em: 23/07/2017 Aceito em: $13 / 06 / 2018$ 